\title{
Image Segmentation Using Two-dimensional Extension of Minimum Within-class Variance Criterion
}

\author{
Fangyan $\mathrm{Nie}^{1}$, Jianqi $\mathrm{Li}^{2}$, Tianyi Tu${ }^{1}$ and Pingfeng Zhang ${ }^{1}$ \\ ${ }^{1}$ College of Computer Science \& Technology, \\ Hunan University of Arts and Science, Changde Hunan 415000, China \\ ${ }^{2}$ Discipline Development Office, \\ Hunan University of Arts and Science, Changde Hunan 415000, China \\ niefyan@163.com
}

\begin{abstract}
Thresholding based on variance analysis of gray levels histogram is a very effective technology for image segmentation. However, its performance is limited in conventional forms. In this paper, a novel method based on two-dimensional extension of within-class variance is proposed to improve segmentation performance. The two-dimensional histogram of the original and local average image is projected to one-dimensional space firstly, and then the minimum within-class variance criterion is constructed for threshold selection. The effectiveness of the proposed method is demonstrated by using examples from the synthetic and real-word images.
\end{abstract}

Keywords: Image thresholding; Two-dimensional histogram; Minimum within-class variance

\section{Introduction}

Image segmentation is fundamental to many image analysis tasks such as object tracking, character recognition, and document analysis, just to name a few [1]. Thresholding is a simple but effective tool for image segmentation [2]. The purpose of this operation is that objects and background are separated into non-overlapping sets. In many applications of image processing, the use of binary image can decrease the computational cost of the succeeding steps compared to using gray-level images. Since image thresholding is a well-researched field, there exist many algorithms for determining an optimal threshold of the image. A survey of thresholding methods and their applications exists in literature [2]. Of which, the variance-based thresholding technology is a kind of famous method for image segmentation [3-9]. In earlier research, Otsu proposed minimum within-class variance criteria to select the best threshold [3]. For every possible threshold value, the method evaluates the goodness of this value if used as the threshold. This evaluation uses either the heterogeneity of both classes or the homogeneity of every class. By minimizing the criterion function, the means of two classes can be separated as far as possible and the variances in both classes will be as minimal as possible. This method still remains one of the most referenced thresholding methods.

Most of the proposed methods selection thresholds which depend solely on the onedimensional (1D) gray level histogram of the image. However, more information contained in the image can be utilized to obtain better segmentation. Since 1D histogram of an image, which only represents the gray level distribution of the image, does not take into consideration the spatial correlation between the pixels in the image, the performance of the 
proposed methods based on 1D histogram might degrade rapidly when the signal-to-noise ratio (SNR) is decreased. For this reason two-dimensional (2D) thresholding methods which employ point pixel information and the local average gray level of the neighborhood pixels have been proposed. Liu and Li [4] proposed a 2D variance-based thresholding method which performs much better than 1D Otsu's method when images are corrupted by noise.

Although the extension of the 1D thresholding method to the 2D histogram results in much better segmentation, it gives rise to the exponential increment of computation. The computation complexity of Liu and Li's method, using an exhaustive search, is bounded by $O\left(L^{4}\right)$, where $L$ is the number of gray levels of image. Gong et al., [5] proposed a fast algorithm which can reduce the computation complexity to $O\left(L^{2}\right)$. But the computation complexity of the method of Otsu (1D method) is only bounded by $O(L)$. In this paper, a novel 2D extension of variance-based thresholding method which decreases the computation complexity of $2 \mathrm{D}$ thresholding from $O\left(L^{2}\right)$ to $O(2 L)$ is introduced. In our new method, the gray levels of the pixels and the local average gray level of the neighborhood pixels form a 2D histogram. However, this 2D parameter space is reduced to a 1D histogram through $1 \mathrm{D}$ projection summation of 2D histogram, while assigning equal weights to both variables of gray levels. Then, the variance-based thresholding method is applied on the obtained 1D histogram. The experiments on artificial images with different levels of noise and real images demonstrate that the proposed approach can perform segmentation well.

\section{Conventional Variance-based Thresholding Method}

Without losing generality, let $I$ denote a gray scale image with $L$ gray levels $G=[0,1$, $\ldots, L-1]$ of size $M \times N, f(x, y)$ be the gray value of the pixel located at the point $(x, y)$ and $x \in\{1,2, \ldots, M\}, y \in\{1,2, \ldots, N\}$. The number of pixels with gray level $i$ is denoted by $n_{i}$ and the total number of pixels by $M \times N$. The probability of gray level $i$ appeared in the image is defined as

$$
p_{i}=\frac{n_{i}}{M \times N}, \quad p_{i} \geq 0, \quad \sum_{i=0}^{L-1} p_{i}=1
$$

Suppose that the pixels in the image are divided into two classes $C_{0}$ and $C_{1}$ by a gray level t; $C_{0}$ is the set of pixels with levels $[0,1, \ldots, t]$, and $C_{1}$ is the set of pixels with levels $[t+1, t+2, \ldots, L-1] . C_{0}$ and $C_{1}$ normally correspond to the object class and the background one, or vice versa. Then the probabilities of the two classes are given by

$$
\omega_{0}=\sum_{i=0}^{t} p_{i}, \quad \omega_{1}=\sum_{i=t+1}^{L-1} p_{i}
$$

The mean gray levels of the two classes can be defined as

$$
\mu_{0}=\sum_{i=0}^{t} i p_{i} / \omega_{0}, \quad \mu_{1}=\sum_{i=t+1}^{L-1} i p_{i} / \omega_{1}
$$

and corresponding class variances are given by

$$
\sigma_{0}^{2}=\sum_{i=0}^{t}\left(i-\mu_{0}\right)^{2} p_{i} / \omega_{0}, \quad \sigma_{1}^{2}=\sum_{i=t+1}^{L-1}\left(i-\mu_{1}\right)^{2} p_{i} / \omega_{1}
$$

the within-class variance in Otsu [3] method is defined by

$$
\sigma_{w}^{2}=\omega_{0} \sigma_{0}^{2}+\omega_{1} \sigma_{1}^{2}
$$


the optimal threshold $t^{*}$ can be determined by

$$
t^{*}=\arg \min _{t \in G}\left[\sigma_{w}^{2}(t)\right]
$$

The performance of $1 \mathrm{D}$ Otsu method might degrade rapidly when the SNR is decreased. For this reason 2D Otsu approaches which employ point pixel information and the local average gray level of the neighborhood pixels have been proposed [4-5].

\section{The Proposed Method}

In this section, a 2D extension of within-class variance criterion is presented. The computation complexity of the new method reduces to $O(2 L)$ from $\mathrm{O}\left(L^{2}\right)$ of fast 2D Otsu method, where $L$ is the number of gray levels in the image. Let $g(x, y)$ denotes the local average gray value in a $w \times w$ neighborhood window, i.e.,

$$
g(x, y)=\left\lfloor\frac{1}{w \times w} \sum_{m=-a}^{a} \sum_{n=-a}^{a} f(x+m, y+n)\right\rfloor
$$

where $a=\lfloor w / 2\rfloor,\lfloor\bullet\rfloor$ denotes the integer part of the number $\bullet, w<\min (M, N)$, in general $w$ is an odd number. Let $n_{i j}$ be the frequency of pair $(i, j)$, where $f(x, y)=i$ and $g(x, y)=j$, then

$$
p_{i j}=\frac{n_{i j}}{M \times N}, \quad 0 \leq i<L, \quad 0 \leq j<L
$$

is a $2 \mathrm{D}$ histogram of image $I$. The $2 \mathrm{D}$ histogram is a matrix of size $L \times L$, which is shown in Figure 1. If we assume that the pair $(s, t)$ is a threshold vector to be used for thresholding, the $(s, t)$ divides $2 \mathrm{D}$ histogram into four quadrants. The intersection of the orthogonal lines produces the overall thresholding point $(s, t)$, as shown in Figure 1(a). These quadrants can be further classified into the diagonal quadrants 0 and 2 and offdiagonal quadrants 1 and 3, respectively, in Figure 1(a). Since two of the quadrants, 1 and 3 , contain information about edges and noise alone, they are ignored in the calculation in traditional 2D histogram thresholding methods, these methods only used two of the quadrants for segmentation, i.e., quadrant 0 pixels as background and quadrant 2 pixels as foreground. However, these methods discarded the pixels located in quadrants 1 and 3, which may ignore important information concerning the objects to be segmented. Sahoo et al., [10] recognized this problem firstly, and then proposed a thresholding line in the 2D histogram plane to provide better segmentation. Such a line, straight line AB is shown in Figure 1(a). However, this method is still a costly method, as there are approximately $4 \times 10^{5}$ lines that bisect a $256 \times 256$ histogram [11]. Based on the idea of Sahoo et al.'s method, some improved methods have been proposed to overcome the defects of time-consuming [11]. 


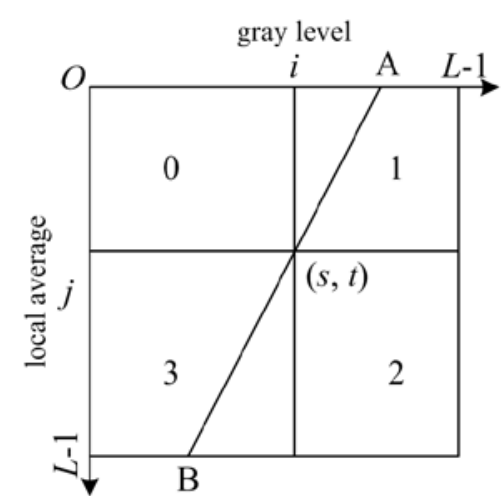

(a) tradition partition method

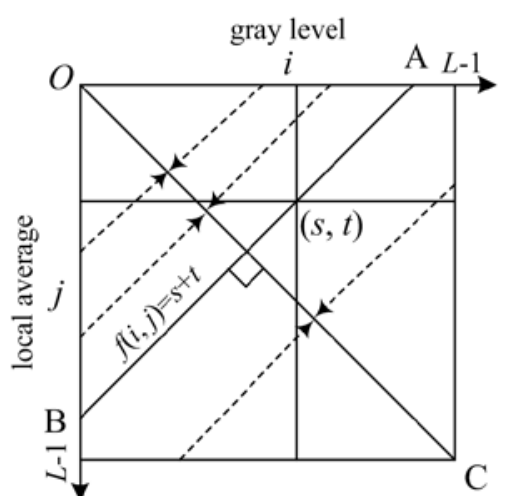

(b) the proposed method

\section{Figure 1. 2D Gray Level Histogram and its Partition}

Based on the idea of thresholding line to divide the 2D histogram, an easier and effective form for dividing the 2D histogram plane is adopted in this paper. As shown in Figure 1(b), passing through $(s, t)$, the line $A B$ is defined to be the line perpendicular to principal diagonal $\mathrm{OC}$ of the $2 \mathrm{D}$ histogram. And then segment the image using line $\mathrm{AB}$ as the optimal thresholding line.

From Figure 1(b), it can be seen that the geometric equation of the line $A B$ is $f(i, j)=s+t$, and $0 \leq f(i, j) \leq 2(L-1)$. If we consider the straight line OC as a projection axis, let us now consider the function $f(i, j)$ as a new variable $r$, and construct its histogram. Obviously, each bin in this histogram will contain a contribution only from a unique line in the two-dimensional gray level histogram matrix, i.e.,

$$
p_{r}=\sum_{r=i+j} p_{i j}, \quad 0 \leq i<L, \quad 0 \leq j<L
$$

Hence, the 2D parameter space was reduced to a 1D histogram of the variable $r$, obviously $r \in\{0,1, \ldots, 2(L-1)\}$. Figure 2 illustrates an example of $1 \mathrm{D}$ projection of 2D histogram. In Figure 2, the image is Lena with size of $512 \times 512$, and the neighborhood window for forming local average image is $5 \times 5$.

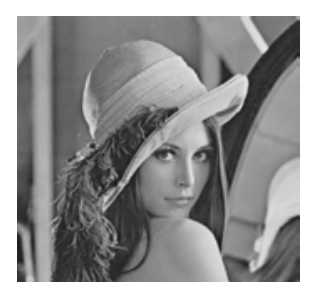

(a) Lena

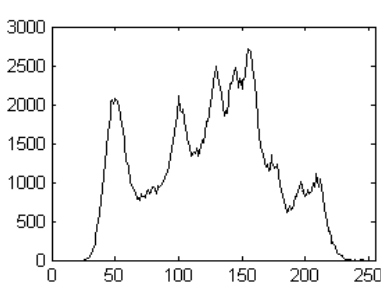

(b) Histogram

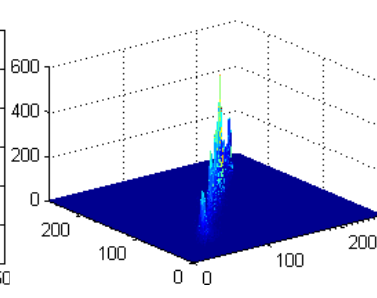

(c) 2D histogram

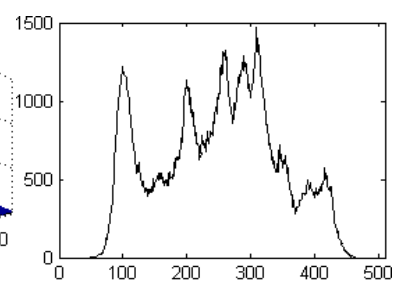

(d) New 1D histogram

Figure 2. An Example of 1D Projection of 2D Histogram

At the new 1D histogram, let $z$ is an assumed threshold value. For image segmentation, If we let

$$
\pi_{0}=\sum_{r=0}^{z} p_{r}, \quad \pi_{1}=\sum_{r=z+1}^{2(L-1)} p_{r}
$$

and 


$$
\begin{aligned}
& m_{0}=\frac{1}{\pi_{0}} \sum_{r=0}^{z} r p_{r}, \quad m_{1}=\frac{1}{\pi_{1}} \sum_{r=z+1}^{2(L-1)} r p_{r} \\
& \sigma_{0}^{2}=\frac{1}{\pi_{0}} \sum_{r=0}^{z}\left(r-m_{0}\right)^{2} p_{r}, \quad \sigma_{1}^{2}=\frac{1}{\pi_{1}} \sum_{r=z+1}^{2(L-1)}\left(r-m_{1}\right)^{2} p_{r}
\end{aligned}
$$
i.e.,

then we can construct the within-class variance criterion on the new $1 \mathrm{D}$ histogram,

$$
\sigma_{w}^{2}=\pi_{0} \sigma_{0}^{2}+\pi_{1} \sigma_{1}^{2}
$$

When $\sigma_{w}^{2}$ is minimized, the value at the new $1 \mathrm{D}$ histogram is considered to be the optimum threshold value. This can be achieved with a cheap computational effort.

$$
z^{*}=\arg \min _{z \in U}\left[\sigma_{w}^{2}(z)\right]
$$

where $U=\{0,1, \ldots, 2(L-1)\}$. From above, if the optimum threshold $z^{*}$ is found, it signifies the optimum threshold line $f(i, j)=i+j=z^{*}$ in Figure 1(b) is found. So in the new method, the pixels in the $\triangle \mathrm{OAB}$ region would be below the threshold $z^{*}$ while the rest would be above it. The result of thresholding an image function $f(x, y)$ at optimal threshold $z^{*}$ is a binary function $\bar{f}(x, y)$ such that

$$
\bar{f}(x, y)= \begin{cases}0, & \text { if }\left(f(x, y)+g(x, y) \leq z^{*}\right) \\ 1, & \text { if }\left(f(x, y)+g(x, y)>z^{*}\right)\end{cases}
$$

where $f(x, y)$ and $g(x, y)$ are the intensities at $(x, y)$ of the original image and the local average image, respectively.

\section{Performance Evaluation and Experiments}

In this section, we present the experimental results obtained via the application of our method to different kinds of images. This section is divided into three parts; in the first subsection, we evaluate the performance results in comparison with of three other methods: Otsu method [3], one method based on two-dimensional Otsu criterion [5] and Li et al., [9] method based on statistical variance analysis theory. The experiments on the real-world images are discussed in the second subsection.

The algorithms are coded in Matlab and run on a $2.80 \mathrm{GHz}$ Pentium(R) Dual-Core CPU personal computer, under Microsoft Window 7 Operating system. In our experiments, when $w=3$, the proposed method can obtain good segmentation results. So, unless otherwise specified, the local neighborhood window $w$ applied to generate 2D histogram is set to 3 .

\subsection{Evaluation of the Performance based on Synthetic Images}

In order to measure the performance of the segmentation, we used the criterion of misclassification error $(M E)$ [2]. $M E$ is defined in terms of correlation of images with human observation. It is the ration of the number of pixels in the background falsely classified in the foreground and vice versa. $M E$ can be simply expressed as

$$
M E=1-\frac{\left|B_{O} \cap B_{T}\right|+\left|F_{O} \cap F_{T}\right|}{\left|B_{O}\right|+\left|F_{O}\right|}
$$


where background and foreground are denoted by $B_{O}$ and $F_{O}$ for the original image, and by $B_{T}$ and $F_{T}$ for the segmented image, respectively. In the best case of ideal thresholding, $M E$ is equal to 0 and, in the worst case, $M E$ value is 1 .

We used a synthetic image with different degrees of noise measured by the standard deviation $\sigma$ of added Gaussian noise. Figure 3(a) is the synthetic image with 256×256 pixels and two intensities (85 and 170). The ground truth of synthetic image is shown in Figure 3(b). Figure 3(c) is an image added Gaussian noise on original synthetic image, whose mean is 0 and variance $\sigma^{2}$ is 900 , i.e., the standard deviation $\sigma$ is 30. Figures 3(d) and 3(e) show the 1D and 2D histogram of the noisy image, respectively. Figure 3(f) shows the $1 \mathrm{D}$ projection of the 2D histogram. Figures 3(g-i) show the segmented results of noisy image by Otsu method, Gong et al., method, Li et al., method and the proposed method. Table 1 lists the results in terms of thresholds, number of misclassified pixels, $M E$ and running time obtained by applying each thresholding method to the noisy image as shown in Figure 3(b).

From Figure 3, it can be seen that the separability of foreground and background at 1D projection histogram is better than the original 1D gray level histogram. From the segmented results in Figure 3, we also can see that the result obtained by the proposed method is better than those obtained by the reference methods. From Table 1, it can be seen that the threshold obtained by the proposed method is 254 (corresponding to 586 misclassified pixels and its $M E$ equals to 0.0089), whereas that by the Otsu method is 127 (corresponding to 5093 misclassified pixels and its $M E$ equals to 0.0777), Gogn et al.'s, fast 2D Otsu method is [154,131] (corresponding to 2152 misclassified pixels and its $M E$ equals to 0.0328), and $\mathrm{Li}$ et al.'s, statistical variance analysis method is 129 (corresponding to 5048 misclassified pixels and its $M E$ equals to 0.0770). Among these thresholding methods, the best result was provided by the proposed method. As can be seen from the thresholded images in Figure 3 and Table 1, one can conclude that the proposed method can provide better results than conventional 1D variance-based thresholding method and Gong et al.'s, fast 2D Otsu method, and the result obtained by Gong et al.'s, method is better than those by 1D methods.

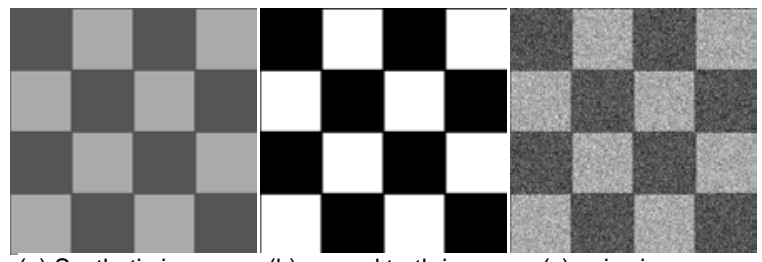

(a) Synthetic image

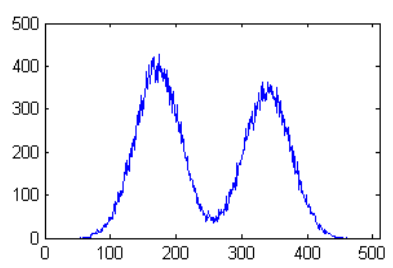

(f) 1D projection histogram

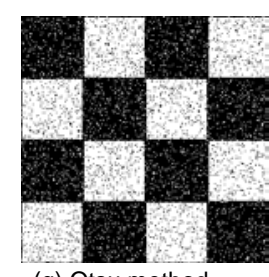

(g) Otsu method

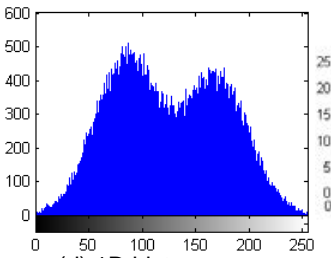

(d) $1 \mathrm{D}$ histogram

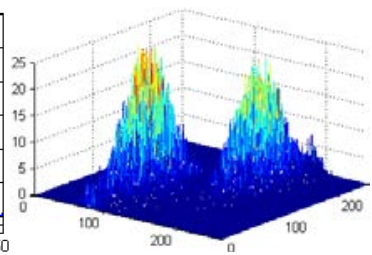

(e) 2D histogram

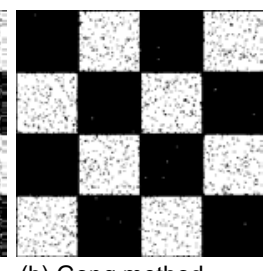

(h) Gong method

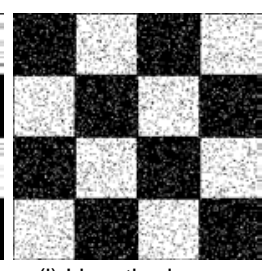

(i) Li method

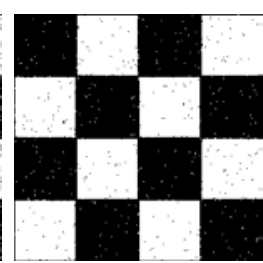

(j) Proposed method

Figure 3. A Synthetic Noisy Image and its Segmented Results by Different Methods 


\section{Table 1. Thresholded Results for the Synthetic Noisy Image of Different Methods}

\begin{tabular}{lcccc}
\hline \multicolumn{1}{c}{ Method } & Otsu method & Gong method & Li method & Proposed method \\
\hline Threshold & 127 & {$[154,131]$} & 129 & 254 \\
Misclassified pixels & 5093 & 2152 & 5048 & 586 \\
$M E$ & 0.0777 & 0.0328 & 0.0770 & 0.0089 \\
Running time (ms) & 31 & 97 & 20 & 46 \\
\hline
\end{tabular}

In order to more fully describe the performance of the proposed method, the experiments on synthetic images added different degrees noise are implemented. The comparison of the results $M E$ provided by the proposed method and the reference methods, based on the segmentation of the synthetic images, with different degrees of noise (Gaussian noise with 0 mean and variance $\sigma^{2}$ ), is presented on Figure 4 . Since the noise is randomly added, we run the simulation 20 times to get mean $M E$ for each magnitude (represented by the standard deviation $\sigma$ of Gaussian noise, and the standard deviation $\sigma$ is gradually increased). From Figure 4, we can clearly see that the proposed method can achieve better performance and lower $M E$ at all magnitude of the noise.

\subsection{Experiments on Real Images}

We have applied the proposed method to a variety of real images. Due to the page limit, only some images are shown here. Figure 5-7 give the thresholding experiments.

Figure 5 shows the Bacteria image and its segmented results. Figure 5(a) is the original Bacteria image with size of $178 \times 178$. Figures $5(\mathrm{~b}-\mathrm{d})$ show the $1 \mathrm{D}$ gray levels histogram, 2D gray levels histogram, and the $1 \mathrm{D}$ projection histogram of the 2D histogram of Bacteria image, respectively. Figures 5(e-h) show the thresholded image of original Bacteria image. From the obtained thresholded results, it can be seen that the result by the proposed method is better than those obtained by other methods.

Figures 6-7 give two thresholding experiments on two noisy images. Figures 6-7(a) are the two original images, one is the Blood1 image with size of $265 \times 272$ and the other is the Eight image with size of $242 \times 308$. Figures 6-7(b) are the images with Gaussian noise and Figures 6-7(c) are their 1D histograms. Figures 6-7(d) show the 1D projection histogram of the noisy images. The image shows in Figure 6(b) is added Gaussian noisy with zero mean and standard deviation 30 and the image show in Figure 7(b) is added Gaussian noisy with zero mean and standard deviation 40. Figures 6-7(e-h) are the segmented results by the methods that are compared with each other. From Figures 6-7, we can see that the experiments on two noisy image show the proposed method performs much better than other methods. 


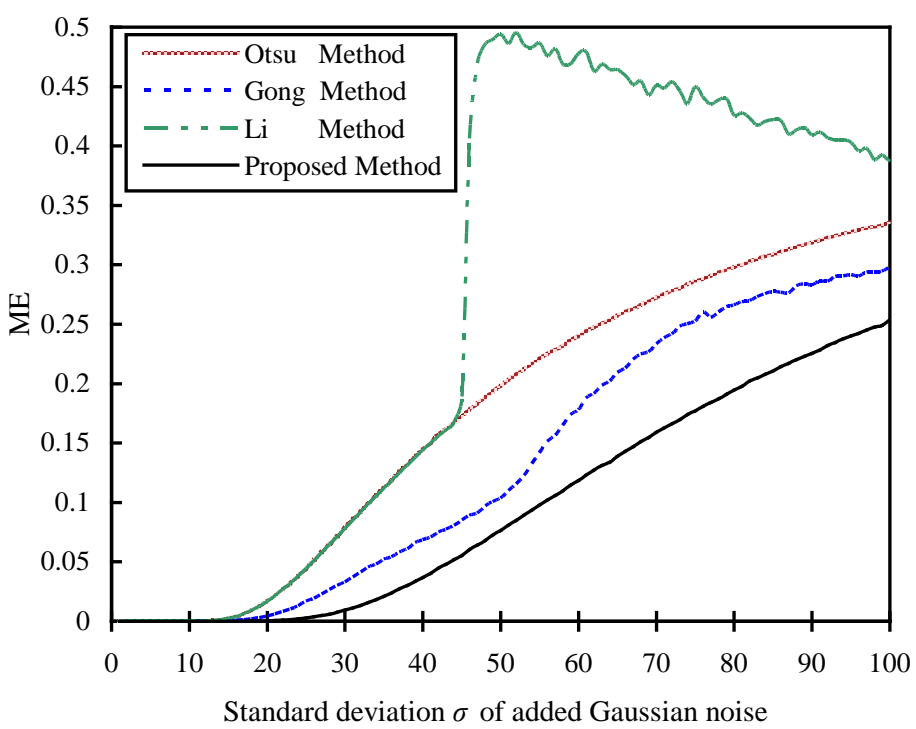

Figure 4. The Relation between ME and the Magnitude of Added Gaussian Noise

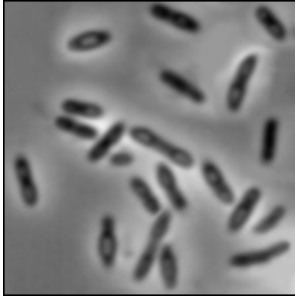

(a) Original image

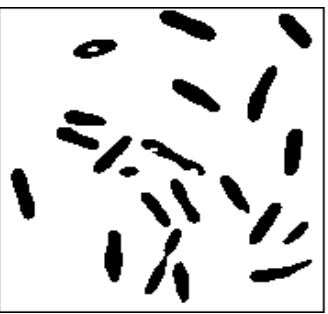

(e) Otsu method

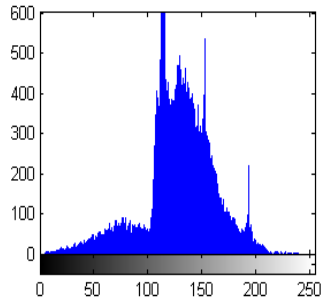

(b) 1D histogram

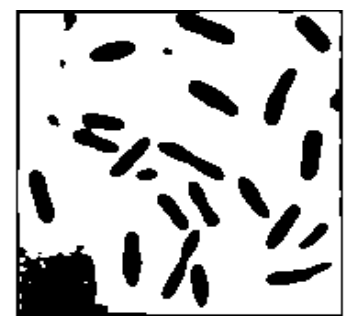

(f) Gong method

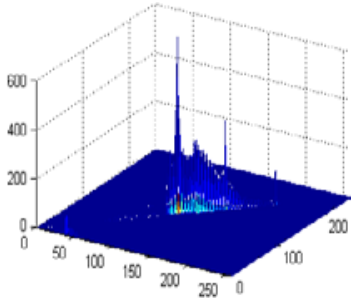

(c) 2D histogram

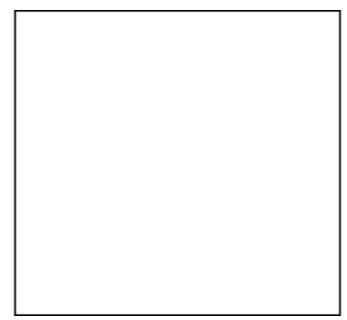

(g) Li method

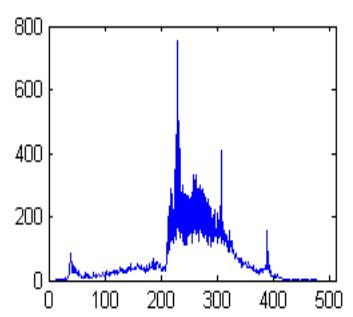

(d) 1D projection histogram

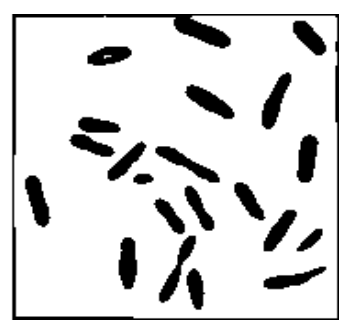

(h) Proposed method

Figure 5. Bacteria Image and its Segmented Results

Table 2 shows the thresholds found by both methods on test images. From Figures 5, 6 and 7, it can be seen that the separability of the different part of test images at 1D projection histogram is better than the original 1D gray level histogram. From Table 2, we can see that the thresholds found by the proposed method are closer to the ideal valley point of the $1 \mathrm{D}$ projection histogram. From the above examples, we can conclude that the proposed method is robust for the test images. The thresholds found by the proposed method can generate better binary images than those obtained by using other methods. Therefore, the proposed method is better than other methods. 


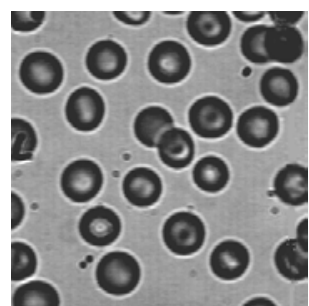

(a) Original image

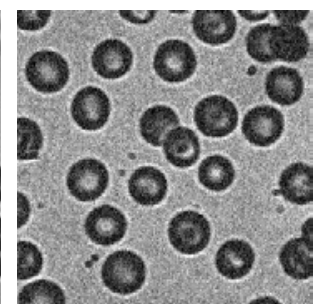

(b) noisy image

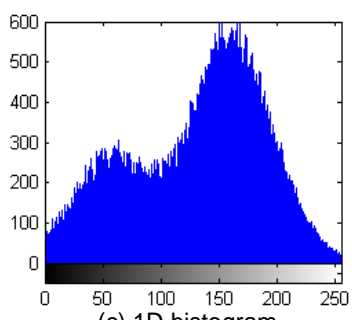

(c) 1D histogram

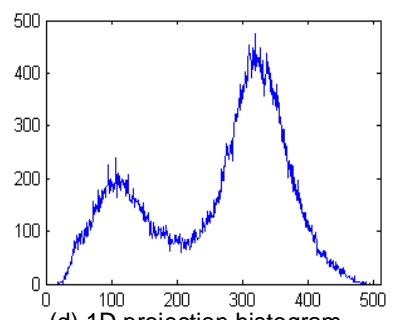

(d) $1 \mathrm{D}$ projection histogram

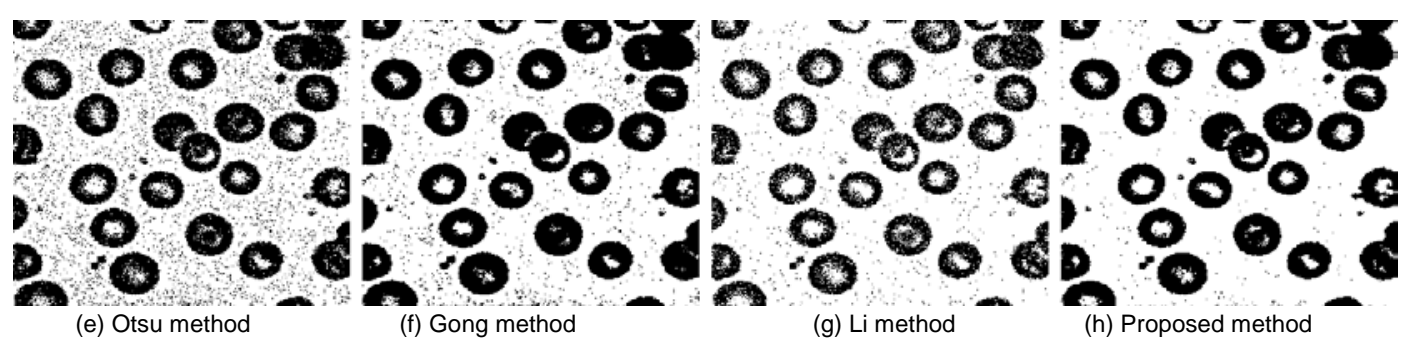

Figure 6. Blood1 Image and its Segmented Results

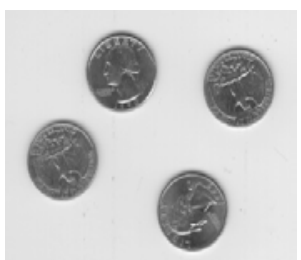

(a) Original image

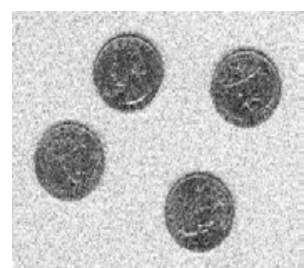

(b) noisy image

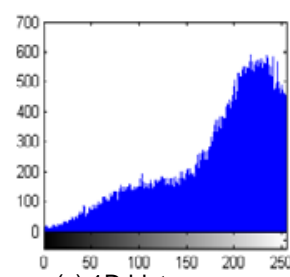

(c) 1D histogram

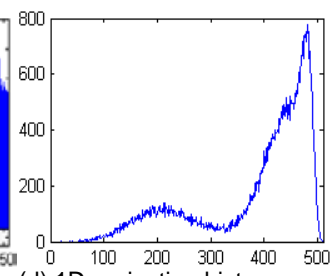

(d) $1 \mathrm{D}$ projection histogram

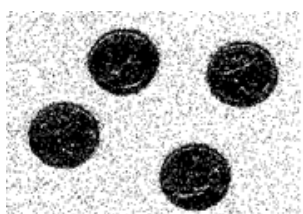

(e) Otsu method

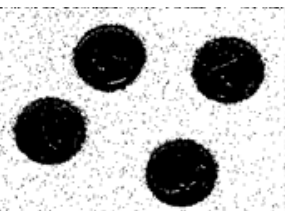

(f) Gong method

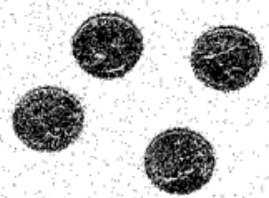

(g) Li method

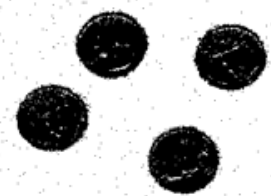

(h) Proposed method

Figure 7. Eight Image and its Segmented Results

Table 2. Thresholds for Test Images by Different Thresholding Methods

\begin{tabular}{ccccc}
\hline Image & Otsu method & Gong method & Li method & Proposed method \\
\hline Bacteria & 96 & {$[119,101]$} & 0 & 202 \\
Blood1 & 114 & {$[124,123]$} & 90 & 224 \\
Eight & 165 & {$[188,171]$} & 134 & 325 \\
\hline
\end{tabular}

Table 3 shows the time required by every method on test image. The computing time was measured in millisecond. From Table 3, we can see that the time required by 1D method, i.e., Otsu method and $\mathrm{Li}$ et al., method, is lower than 2D histogram thresholding methods. Since the computational complexity of the $1 \mathrm{D}$ histogram thresholding method is $O(L)$, where $L$ is the number of the gray levels of image. The computational complexity of Gong et al., method is $O\left(L^{2}\right)$, therefore, the computational cost is the highest. The computational complexity of the proposed method is $O(2 L)$, 
therefore, the time required is higher than 1D methods and lower than Gong et al., method.

Table 3. The Computational Cost (ms) of each Methods on Test Images

\begin{tabular}{ccccc}
\hline Image & Otsu method & Gong method & Li method & Proposed method \\
\hline Bacteria & 35 & 124 & 22 & 57 \\
Blood1 & 37 & 137 & 23 & 65 \\
Eight & 34 & 133 & 23 & 67 \\
\hline
\end{tabular}

\section{Conclusions}

An automatic thresholding of gray level image using minimum within-class variance criterion based on 1D projection of 2D histogram is proposed. The proposed method is based on the 2D gray level histogram of images, which considers both the local information as well as the pixel intensity. The experiments prove that the obtained threshold can be applied to perform image thresholding. The thresholding results are compared with those from conventional variance-based methods. The experimental results prove that the proposed method outperforms conventional variance-based methods. The proposed approach can have wide application in computer vision and image processing.

\section{Acknowledgements}

The authors wish to thank the anonymous referees for the valuable comments and suggestions which helped to improve this paper. This research is partially supported by the Young Core Instructor Foundation of Hunan Provincial Institutions of Higher Education of China and the Doctor Scientific Research Startup Project Foundation of Hunan University of Arts and Science, China.

\section{References}

[1] N. Pal and S. K. Pal, “A review on image segmentation techniques”, Pattern Recognition, vol. 26, no. 9, (1993), pp. 1277-1294.

[2] M. Sezgin and B. Sankur, "Survey over image thresholding techniques and quantitative performance evaluation”, Journal of Electronic Imaging, vol. 13, no. 1, (2004), pp. 146-165.

[3] N. Otsu, "A threshold selection method from gray-level histograms", IEEE Transactions on Systems, Man, and Cybernetics, vol. 9, no. 1, (1979), pp. 62-66.

[4] J. Liu and W. Li, "The automatic thresholding of gray-level pictures via two-dimensional Otsu method", Acta Automatica Sinica, vol. 19, no. 1, (1993), pp. 101-105.

[5] J. Gong, L. Li and W. Chen, "Fast recursive algorithms for two-dimensional thresholding", Pattern Recognition, vol. 31, no. 3, (1998), pp. 295-300.

[6] I. Hannah, D. Patel and R. Davies, "The use of variance and entropic thresholding methods for image segmentation”, Pattern Recognition, vol. 28, no. 8, (1995), pp. 1135-1143.

[7] Z. Hou, Q. Hu and W. Nowinski, "On minimum variance thresholding”, Pattern Recognition Letters, vol. 27, no. 14, (2006), pp. 1732-1743.

[8] Y. Qian, Q. Hu, S. Luo and W. Nowinski, “Thresholding based on variance and intensity contrast”, Pattern Recognition, vol. 40, no. 2, (2007), pp. 596-608.

[9] Z. Li, C. Liu, G. Liu, Y. Cheng, X. Yang and C. Zhao, "A novel statistical image thresholding method”, AEU-International Journal of Electronics and Communications, vol. 64, no. 12, (2010), pp. 1137-1147.

[10] P. Sahoo, D. Slaaf and T. Albert, "Thresholding selection using a minimal histogram entropy difference”, Optical Engineering, vol. 36, no. 7, (1997), pp. 1976-1981.

[11] E. Jansing, T. Albert and D. Chenoweth, “Two-dimensional entropic segmentation”, Pattern Recognition Letters, vol. 20, no. 3, (1999), pp. 329-336. 


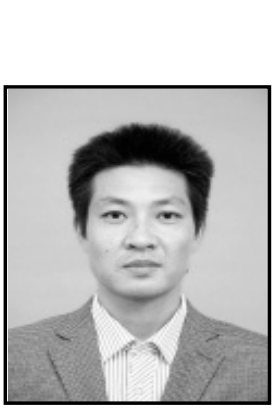

\section{Authors}

Fangyan Nie was born in 1977 and graduated from National University of Defense Technology, Changsha, China, in 1999. He received his M.S. degree in Computer Science and Technology from Guizhou University, Guiyang, China, in 2005 and the Ph.D. degree in Instrument Science and Technology from Chongqing University, Chongqing, China, in 2010. His research interests include image processing, pattern recognition and intelligent optimization algorithms. He has published more than 20 papers in international/national journals.

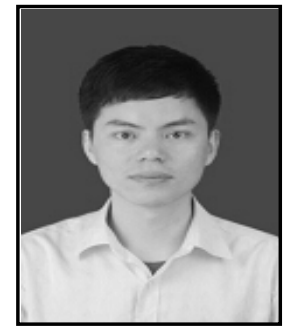

Jianqi Li was born in 1980 and graduated from Central South University, Changsha, China, in 2002. He received his M.S. degree from Hunan University, Changsha, China, in 2007 and the Ph.D. degree from Central South University, Changsha, China, in 2013. His research interests include image processing, pattern recognition and complex industrial process modeling and optimization. He has published more than 20 papers in international/national journals.

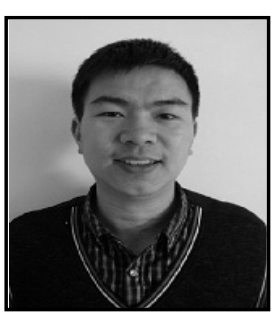

Tianyi Tu was born in 1980 and graduated from Xiangtan University, Xiangtan, China in 2002. He received his M.S. degree in Computer Applications Technology from Xiangtan University, Xiangtan, China, in 2005. His research interests include image processing, pattern recognition and intelligent optimization algorithms.

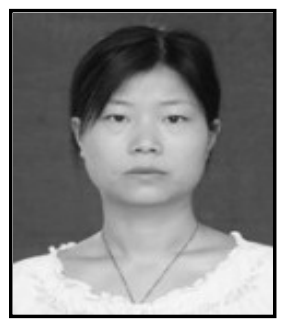

Pingfeng Zhang was born in 1980 and graduated from Hunan University of Arts and Science, Changde, China, in 2008. Her research interests include image and information processing. 
International Journal of Signal Processing, Image Processing and Pattern Recognition Vol.6, No.5 (2013) 